\title{
PERFIL FARMACOTERAPÊUTICO ADJUVANTE DE PACIENTES ONCOLÓGICOS DE UMA CASA DE ACOLHIMENTO NO INTERIOR DA BAHIA
}

\author{
ADJUVANT PHARMACOTERAPEUTICAL PROFILE OF ONCOLOGICAL \\ PATIENTS FROM A WELCOMING HOUSE INSIDE THE BAHIA
}

\section{Phydel Palmeira Carvalho, Érika Pereira De Souza, Gladistone Correia Messias, Maria José Santos Viana Fontoura, Kelle Oliveira Silva}

Universidade Federal da Bahia - UFBA

\begin{abstract}
Cancer is one of the most important public health problems worldwide and is among the main causes of death. The purpose of this study is to outline the adjuvant pharmacotherapeutic profile in the palliative support of cancer patients in a nursing home in the city of Vitória da Conquista Bahia. Twenty-seven individuals over 40 years of age were interviewed, the variables studied were socioeconomic, comorbidities and palliative medication prescriptions. The research was performed during the months of October to December 2014. The most frequent signs and symptoms were weakness (96\%), pain (70\%), constipation (67\%) and insomnia (67\%). The main adjuvant treatments were treatment of cancer pain (30.7\%) and inhibition of emesis (28.4\%). It is concluded that palliative pharmacotherapeutic support was used in the treatment of cancer patients, with prevalence of drugs for pain, nausea and emesis control, as well as prophylaxis of ulcerations.
\end{abstract}

Key words: Adjuvant pharmacotherapeutic; Palliative Care; Drug Utilization; Quality of Life.

\section{Resumo}

O câncer é um dos mais importantes problemas de saúde pública mundial e está entre as principais causas de óbito. Este estudo tem por objetivo traçar o perfil farmacoterapêutico adjuvante no suporte paliativo de pacientes oncológicos em uma casa de acolhimento no município de Vitória da Conquista - Bahia. Foram entrevistados 27 indivíduos acima de 40 anos, as variáveis estudadas foram socioeconômicas, comorbidades e prescrições medicamentosas paliativas. A pesquisa foi realizada durante os meses de outubro a dezembro de 2014. Os sinais e sintomas mais frequentes foram fraqueza (96\%), dor (70\%), constipação (67\%) e insônia (67\%). Os principais tratamentos adjuvantes foram o tratamento da dor oncológica $(30,7 \%)$ e inibição da êmese (28,4\%). Conclui-se que o suporte farmacoterapêutico paliativo foi utilizado no tratamento de pacientes oncológicos, com prevalência dos medicamentos para $o$ controle da dor, da náusea e da êmese, bem como para a profilaxia das ulcerações.

Palavras chave: Farmacoterapia adjuvante; Cuidados paliativos; Uso de medicamentos; Qualidade de Vida. 
Introdução

O câncer é uma doença caracterizada pela multiplicação e disseminação descontrolada de formas anômalas de células do corpo $^{1,2}$. De acordo com estimativas mundiais do projeto Globocan da Agência Internacional para Pesquisa em Câncer (IARC, do inglês International Agency for Research on Cancer) da Organização Mundial da Saúde (OMS), houve 14,1 milhões de casos novos de câncer e um total de 8,2 milhões de mortes por câncer em todo o mundo, em 2012. A incidência de câncer continuará aumentando nos países em desenvolvimento e crescerá ainda mais em países desenvolvidos se medidas preventivas não forem amplamente aplicadas. A estimativa para o Brasil, biênio 2016-2017, aponta a ocorrência de cerca de 600 mil casos novos de câncer. Excetuando-se o câncer de pele não melanoma (aproximadamente 180 mil casos novos), ocorrerão cerca de 420 mil casos novos de câncer ${ }^{3}$.

Vários aspectos devem ser considerados para um efetivo tratamento e possível cura, como o diagnóstico precoce e os meios de reabilitação física, social e psicológica, constituindo como importante incentivo à luta contra esta doença. O impacto de um possível diagnóstico, a confirmação da doença e do seu tratamento influi diretamente no estilo de vida do indivíduo ${ }^{4}$.

Por ser uma doença de caráter invasivo e evolutivo, o câncer apresenta algumas complicações e comorbidades, além dos efeitos colaterais pelo tratamento antineoplásico (cirúrgico, quimioterápico, radioterápico ou associação destes) ou ainda, possíveis reações adversas do tratamento paliativo que causam sofrimento ao paciente ${ }^{2,5}$.

Entre os estudos usados como referência para a compreensão das principais comorbidades ou reações adversas frequentes em pacientes durante o tratamento do câncer estão dor, fadiga, inflamação, náuseas, vômitos, constipação, dispepsias, úlceras gástricas, diarreia, inapetência, desidratação, pressão alta, falta de apetite, dispneia, alterações no sono, ansiedade, depressão, falta de ar, xerostomia, prurido, icterícia, sudorese, alopecia, astenia, edema, mal-estar geral, vertigem, cefaleia, nervosismo, sedação, hipertermia, diurese sanguinolenta, disúria, tosse, dor na micção, entre outros ${ }^{4,5,6,8,12,13}$. Todos diminuem de algum modo a qualidade de vida, merecendo, portanto, a atenção dos profissionais de saúde ${ }^{1}$ para 0 tratamento dessas alterações.

Nas fases iniciais do câncer, com objetivo de cura ou remissão, o tratamento geralmente é agressivo. Quando a doença já se apresenta em estágio avançado ou evolui para esta condição mesmo durante o tratamento com intenção curativa, a abordagem paliativa deve começar a ser utilizada no manejo dos sintomas de difícil controle e de alguns aspectos psicossociais associados à doença. Na fase terminal, com o pouco tempo de vida do paciente, o tratamento paliativo se impõe para garantir qualidade de vida $^{5,6}$.

O tratamento das comorbidades pode ser realizado através de três meios, como o farmacológicos (analgésicos não opioides ou opioides, antieméticos, antiulcerosos, laxantes, fármacos que atuam no Sistema Nervoso Central (SNC), entre vários outros medicamentos adjuvantes), não farmacológicos (intervenção psicológica, física ou radioterapia) e invasivos (bloqueio nervoso, cirurgia paliativa, neurocirurgia) $)^{5,8}$. Desta forma, o objetivo do estudo foi traçar o perfil farmacoterapêutico adjuvante no suporte paliativo de pacientes oncológicos em uma casa de acolhimento no município de Vitória da Conquista - Bahia.

\section{Metodologia}

Para a realização desta pesquisa, segue um estudo quali-quantitativo do tipo transversal ${ }^{9,10}$. A pesquisa foi realizada durante os meses de outubro a dezembro de 2014, no município de Vitória da Conquista. A coleta de dados foi realizada na Casa de Acolhimento ao Paciente Oncológico do Sudoeste da Bahia (Casa do Amor/CAPOS) em Vitória da Conquista - BA.

Foram realizadas 27 entrevistas com pessoas de ambos os sexos, sendo os critérios de exclusão os pacientes menores de 18 anos e os que não conseguiram responder a entrevista por estado debilitado e, como critérios de inclusão, pessoas de ambos os sexos, maiores de 18 anos em situação de acolhimento, capaz de ser entrevistado e apresentando algum tipo de câncer já diagnosticado em acompanhamento médico.

Para a coleta de dados, foi utilizado um formulário, contendo questões subjetivas organizadas com dados pessoais referentes ao aspecto sociodemográfico, dados clínicos relacionados à condição de saúde e comorbidade. Foram avaliadas também as prescrições medicamentosas dos últimos 7 dias, conforme o prontuário do paciente. Em seguida, os dados foram tabulados em dupla digitação utilizando o programa MS Office Excel 2010 e apresentados em forma de porcentagem. 
O estudo foi aprovado pelo Comitê de Ética e Pesquisa da Faculdade Independente do Nordeste (FAINOR), sob Protocolo CAAE no 34561414.1.0000.5578. Todos os participantes foram informados a respeito dos objetivos do estudo e motivos da pesquisa, sendo garantido durante a entrevista o sigilo e anonimato aos participantes através da assinatura do Termo de Consentimento Livre e Esclarecido.

\section{Resultados}

Para a realização desta pesquisa, foram abordados vinte e nove (29) pacientes oncológicos em uma casa de acolhimento, sendo que, dois (02) desses pacientes estavam debilitados e sem condições para realizar o estudo, totalizando vinte e sete (27) entrevistados.

O perfil sociodemográfico está expresso na Tabela 1. Sendo uma população composta principalmente por idosos, não alfabetizados, com baixa renda familiar e proveniente de outras localidades.

Em relação ao diagnóstico (Tabela 2), os tipos de câncer mais frequentes foram próstata $(26 \% ; n=07)$ e mama $(14 \% ; n=04)$. Todos os pacientes se submeteram a algum tipo de esquema de tratamento antineoplásico. A análise demonstrou que a maioria dos pacientes havia feito a associação de radioterapia e quimioterapia (34\%; n=09) (Tabela 2).

O tratamento para o câncer evoluiu consideravelmente nas últimas décadas, consistindo em cirurgias diversas, as quais podem estar associadas a outras modalidades como quimioterapia, radioterapia e hormonioterapia. Devido a seus efeitos adversos, o tratamento é considerado, muitas vezes, mutilador, podendo provocar modificações na autoimagem, perda da capacidade produtiva; desempenho profissional ou doméstico e, consequentemente, alterações psicológicas, emocionais e sociais ${ }^{11}$.

Quanto aos sinais e sintomas apresentados durante a entrevista, com mais de uma resposta por paciente, os relatos com maior frequência foram, fraqueza e fadiga $(96 \% ; n=26)$, dor $(70 \%$; $n=19)$, constipação (67\%; $n=18)$ e insônia $(67 \%$; $n=18$ ) (Tabela 3).

Os sinais e sintomas apresentados mostram - que também foi descrito por outros estudos $^{6,8,12,13,14,15,16,18,21,22,24}$. A fraqueza e a dor são manifestações frequentes no paciente oncológico, sendo considerados os sintomas mais prevalentes e incapacitantes nessa população ${ }^{6,12,13}$. Portanto, causa um grande desconforto no paciente, significando agravamento do prognóstico ou êxito letal, diminuição da autonomia, do bem-estar e da qualidade de vida ${ }^{4,5}$.

Tabela 1 - Distribuição dos pacientes oncológicos segundo dados sócio demográficos ( $\mathrm{N}=27$ ).

\begin{tabular}{|c|c|c|}
\hline Característica & $N$ & Percentual \\
\hline \multicolumn{3}{|l|}{ Gênero (sexo) } \\
\hline Masculino & 16 & $59 \%$ \\
\hline Feminino & 11 & $41 \%$ \\
\hline \multicolumn{3}{|l|}{ Faixa etária } \\
\hline Menos que 40 anos & Nenhum & $0 \%$ \\
\hline 41 a 50 anos de idade & 05 & $19 \%$ \\
\hline 51 a 60 anos de idade & 03 & $11 \%$ \\
\hline 61 a 70 anos de idade & 08 & $30 \%$ \\
\hline 71 a 80 anos de idade & 06 & $22 \%$ \\
\hline 81 a 90 anos de idade & 03 & $11 \%$ \\
\hline Acima de 91 anos de idade & 02 & $7 \%$ \\
\hline \multicolumn{3}{|l|}{ Renda em salários mínimos* } \\
\hline Sem renda declarada & 01 & $4 \%$ \\
\hline Até 1 salário mínimo & 19 & $70 \%$ \\
\hline 1 a 2 salários mínimos & 07 & $26 \%$ \\
\hline Maior que 2 salários mínimos & Nenhum & $0 \%$ \\
\hline \multicolumn{3}{|l|}{ Escolaridade } \\
\hline Não alfabetizado & 16 & $59 \%$ \\
\hline Ensino Fundamental incompleto & 06 & $22 \%$ \\
\hline Ensino Fundamental completo & 05 & $19 \%$ \\
\hline \multicolumn{3}{|l|}{ Procedência } \\
\hline Vitória da Conquista - BA & Nenhum & $0 \%$ \\
\hline Outros municípios vizinhos & 27 & $100 \%$ \\
\hline
\end{tabular}

*Salário mínimo em 2014: R\$ 724,00 pelo Decreto no 8.166/2013. 
Tabela 2 - Distribuição dos pacientes pelo tipo de neoplasia e o tratamento segundo o diagnóstico ( $\mathrm{N}=27)$.

\begin{tabular}{lll}
\hline Característica & $\boldsymbol{n}$ & Percentual \\
\hline Tipo de neoplasia & & $\mathbf{2 6 \%}$ \\
Próstata & 07 & $\mathbf{1 4 \%}$ \\
Mama & 04 & $\mathbf{1 1 \%}$ \\
Pele & 03 & $\mathbf{1 1 \%}$ \\
Esôfago & 03 & $\mathbf{7 \%}$ \\
Útero & 02 & $\mathbf{7 \%}$ \\
Estômago & 02 & $\mathbf{4 \%}$ \\
Coluna & 01 & $\mathbf{4 \%}$ \\
Pulmão & $\mathbf{4 \%}$ \\
Faringe & 01 & $\mathbf{4 \%}$ \\
Intestino & 01 & $\mathbf{4 \%}$ \\
Vagina/Vulva & 01 & $\mathbf{4 \%}$ \\
Reto & 01 & $\mathbf{3 4 \%}$ \\
Rratamento antineoplásico & 01 & $\mathbf{2 2 \%}$ \\
Radioterapia e Quimioterapia & & $\mathbf{1 5 \%}$ \\
Somenterapia, Quimioterapia e Cirurgia & 09 & $\mathbf{1 1 \%}$ \\
Quimioterapia e Cirurgia & 06 & $\mathbf{7 \%}$ \\
Radioterapia e Cirurgia & 04 & $\mathbf{7 \%}$ \\
Somente Cirurgia & 03 & $\mathbf{4 \%}$ \\
Somente Quimioterapia & 02 & \\
\hline
\end{tabular}

Tabela 3 - Distribuição dos pacientes oncológicos segundo os sinais e sintomas referidos ( $N=27)$.

\begin{tabular}{lll}
\hline Característica & $\boldsymbol{n}$ & Percentual \\
\hline Sinais e sintomas relatados & 26 & $\mathbf{9 6 \%}$ \\
Fraqueza/Fadiga & 19 & $\mathbf{7 0 \%}$ \\
Dor & 18 & $\mathbf{6 7 \%}$ \\
Constipação & 18 & $\mathbf{6 7 \%}$ \\
Insônia & 15 & $\mathbf{5 5 \%}$ \\
Alterações urinárias & 12 & $\mathbf{4 4 \%}$ \\
Perda de apetite & 10 & $\mathbf{3 7 \%}$ \\
Náusea & 10 & $\mathbf{3 7 \%}$ \\
Boca seca (xerostomia) & $\mathbf{3 3 \%}$ \\
Gastrite & 09 & $\mathbf{3 0 \%}$ \\
Vômito & 08 & $\mathbf{3 0 \%}$ \\
Alopecia & 08 & $\mathbf{3 0 \%}$ \\
Tosse & 08 & $\mathbf{2 2 \%}$ \\
Dor durante micção (pós radioterapia) & 06 & $\mathbf{1 9 \%}$ \\
Cefaleia & 05 & $\mathbf{1 5 \%}$ \\
Manchas na pele & 04 & $\mathbf{1 1 \%}$ \\
Prurido & 03 & $\mathbf{1 1 \%}$ \\
Falta de ar & 03 & $\mathbf{1 1 \%}$ \\
Desidratação & 03 & $\mathbf{7 \%}$ \\
Edema & 02 & $\mathbf{7 \%}$ \\
Sudorese & 02 & $\mathbf{7 \%}$ \\
Falta de ar & 02 &
\end{tabular}

A partir do estudo de Agra et al. (2013) ${ }^{14}$ que investigou sobre a constipação intestinal em pacientes com doença oncológica avançada, sendo um dos principais sintomas apresentados pelos entrevistados. A constipação apesar de ser uma manifestação relativamente comum em pacientes que utilizam opióides é agravada por vários outros fatores como desnutrição, desidratação e uso concomitante de outros medicamentos $^{12,15}$.
Os distúrbios de sono, em especial a insônia, são frequentes em paciente com câncer. O diagnóstico do câncer é uma condição aversiva e, a insônia casualmente é vista como um sintoma secundário à depressão ou à ansiedade, com redução do tempo total de sono, sono perturbado, calor, ocorrência de dores, ronco alto e tosse ${ }^{13}$.

O câncer pode acarretar efeito devastador na vida de quem o recebe, pois, mesmo com os 
avanços tecnológicos, ainda é sinônimo de morte, dor e sofrimento. Por isso é fundamental avaliar a presença de sintomatologia ansiosa e depressiva em pacientes com câncer. A importância desse diagnostico em pacientes sob cuidados paliativos deve-se ao fato de que, além do comprometimento emocional, os pacientes deprimidos também apresentam sintomas que são difíceis de "paliar" e que podem ser facilmente aliviados quando tratada adequadamente, com a consequente melhoria da qualidade de vida ${ }^{2,17}$.
Quanto ao perfil farmacoterapêutico, foi observado o uso de medicamentos adjuvantes por $59 \%(n=16)$ e $41 \%$ não fizeram o uso nos últimos 7 dias $(n=11)$. De acordo com a análise das prescrições, foram observados medicamentos para o tratamento da dor oncológica, inibidores da êmese, antiulcerosos, laxantes e fármacos que atuam no SNC (Tabela 4). Com relação as classes representadas no estudo o número de medicamentos adjuvantes mais utilizados, verificou-se uma média de 11,12 por paciente ao longo dos sete dias que antecederam a entrevista.

Tabela 4 - Distribuição da qualidade de vida dos pacientes oncológicos segundo as preocupações relatadas ( $\mathrm{N}=27$ ).

\begin{tabular}{|c|c|c|}
\hline Qualidade de Vida & $n$ & Percentual \\
\hline \multicolumn{3}{|l|}{ Social } \\
\hline Boa & 24 & $89 \%$ \\
\hline Regular & 3 & $11 \%$ \\
\hline Ruim & 0 & $0 \%$ \\
\hline \multicolumn{3}{|l|}{ Físico } \\
\hline Boa & 7 & $26 \%$ \\
\hline Regular & 7 & $26 \%$ \\
\hline Ruim & 13 & $48 \%$ \\
\hline \multicolumn{3}{|l|}{ Meio ambiente } \\
\hline Boa & 27 & $100 \%$ \\
\hline Regular & 0 & $0 \%$ \\
\hline Ruim & 0 & $0 \%$ \\
\hline \multicolumn{3}{|l|}{ Psicológica } \\
\hline Boa & 15 & $56 \%$ \\
\hline Regular & 10 & $37 \%$ \\
\hline Ruim & 2 & $7 \%$ \\
\hline
\end{tabular}

A Organização Mundial da Saúde incentiva que o tratamento ativo e o tratamento paliativo não são mutuamente excludentes e propõe que muitos aspectos dos cuidados paliativos devem ser aplicados mais cedo, no curso da doença, em conjunto com o tratamento oncológico ativo ${ }^{1,7}$.

A farmacoterapia adjuvante tem como objetivo minimizar o desconforto causado pelo câncer, com objetivo de aumentar a analgesia (analgésicos, corticosteroides e anticonvulsivantes/antidepressivos), controlar as reações adversas dos opiáceos e quimioterápicos (os antieméticos, antiulcerosos e laxativos) e manter o controle dos sintomas que estão contribuindo para a dor do paciente, como ansiedade, insônia e depressão (ansiolíticos e antidepressivos) $)^{5}$.

A dor leve costuma ser tratada com analgésicos não esteroidais, como foi apresentado em maioria no estudo. O uso desses medicamentos deve ser reservado aos pacientes com baixo risco de sangramento gastrintestinal ${ }^{19}$. Por ser um dos tratamentos mais utilizados durante a terapia oncológica, pode ser instituído desde o diagnóstico e durante todo o curso da doença, visando o fornecimento de maior conforto ao paciente pelo alívio de sintomas e, especialmente, da dor e inflamação. No entanto, possuem efeitos indesejáveis que podem comprometer o sucesso terapêutico ${ }^{6,20,21}$.

A dor moderada é tratada com associação de paracetamol e codeína, um opióide fraco. O tramadol também é bastante empregado e seu uso possui menor efeito obstipante, mas pode causar náuseas, vômitos, sudorese e tonturas, sendo que, em altas doses, pode causar convulsões $^{6,21}$. A morfina e seus derivados, considerados opióides fortes, é o de primeira linha e promove analgesia adequada para muitos pacientes, mas para outros apresenta efeitos colaterais intoleráveis ou alívio inadequado. Pode causar depressão respiratória, vômito por estimulação da zona quimiorreceptora, inibe mobilidade de músculo liso intestinal e diminui o peristaltismo $^{21}$. Propõe-se o uso de analgésicos, opioides fracos e opioides fortes, nesta sequência. A estes analgésicos podem ser associados fármacos adjuvantes como os 
antidepressivos amitriptilina, sertralina, fluoxetina e anticonvulsivantes como a fenitoína, apresentadas no estudo ${ }^{18}$.

O uso de antieméticos, laxantes e antiulcerosos comumente estão relacionados à terapia quando há complicação da quimio/radioterapia ou quando o paciente é submetido a tratamentos secundários adjuvantes, quando induz efeitos adversos, o que pode trazer melhora nos pacientes quanto às suas complicações gastrointestinais desencadeadas pelos tratamentos ${ }^{20,22}$.

Se ocorrer a falta de controle das náuseas e dos vômitos, certamente irá ocorrer a redução do apetite e o aumento da frequência com que os sintomas aparecem ${ }^{1}$. Para a antiemese profilática, a recomendação é o uso de dexametasona combinados com antagonistas de receptores de serotonina, anterior à quimioterapia $^{23}$. A metoclopramida, também tem a capacidade de diminuir a sensibilidade dos nervos aferentes viscerais que transmitem impulsos a partir do trato gastrintestinal para o centro do vômito, podendo inclusive ser utilizada como antiemético nos casos de êmese refratária ${ }^{23}$, uma possível explicação para o seu maior uso neste estudo.

O tratamento profilático precoce com supressores ácidos da dispepsia para cicatrização temporária das úlceras deve partir da orientação e o periódico acompanhamento. Dentre os utilizados estão os inibidores de bomba de prótons, os bloqueadores $\mathrm{H}_{2}$ e os antiácidos, sendo que a maioria desses foi apresentada no estudo $^{24}$.

Para o tratamento da constipação, incluem o uso de laxantes, supositórios de glicerina, enemas de fosfato de sódio e remoção manual das fezes. Os medicamentos podem ser classificados em grupos, como laxantes osmóticos (lactulose); laxantes salinos (sais de magnésio); laxantes lubrificantes (vaselina líquida) e solução glicerinada; laxantes surfactantes (docusato de sódio); laxantes retais (supositórios de glicerina e enemas de fosfato de sódio) ${ }^{14}$, sendo os esquemas usados nos tratamentos do estudo.

Dos medicamentos que atuam no Sistema Nervoso Central mais utilizados com caráter antidepressivo descritos pela literatura durante o tratamento do câncer são a fluoxetina, paroxetina e sertralina ${ }^{16,17}$. As evidências disponíveis sugerem fortemente que a depressão em pacientes com câncer responde aos antidepressivos tricíclicos (amitriptilina e imipramina) e aos antidepressivos tetracíclicos (mirtazapina) $^{2,16,17}$. Concordando com a maioria dos resultados apresentados na entrevista onde são usados a amitriptilina, sertralina, fluoxetina e mirtazapina.

O tratamento mais utilizado para insônia em pacientes com câncer inclui a prescrição de medicamentos como os benzodiazepínicos (diazepam, midazolam, clonazepam e lorazepam), os benzodiazepínicos agonistas e antidepressivos. Em pessoas com câncer, a insônia é geralmente tratada com essas drogas hipnóticas e apresenta resultados efetivos em curto prazo. No entanto, a utilização prolongada de medicamentos resulta em consequências negativas como a dependência e a tolerância. Diante disso, os estudos de intervenções não medicamentosas para o manejo da insônia estão sendo ampliados ${ }^{13}$. Para minimizar as reações adversas a antidepressivos e anticonvulsivantes, o esquema terapêutico deve-se iniciar com baixas doses e aumentar gradativamente ${ }^{6}$.

Por isso, o adequado controle das comorbidades durante o tratamento do câncer é tema atual e considerado indicador de qualidade de vida e de assistência e é importante dentro do contexto da promoção da saúde $e^{6,25}$.

\section{Conclusão}

Esta pesquisa possibilitou verificar que entre a farmacoterapia adjuvante mais usada durante o tratamento do câncer, houve prevalência dos medicamentos para o controle da dor, da náusea e da êmese, bem como para a profilaxia das ulcerações. Por último, os fármacos que atuam no SNC, a fim de controlar transtornos como a ansiedade e insônia.

Desta forma, conclui-se que o fato de o indivíduo passar pela experiência de ter câncer e buscar tratamentos paliativos com fármacos adjuvantes merece uma atenção especial. Os resultados encontrados nessa pesquisa ajudam a fortalecer os dados presentes na literatura, onde mostra a importância de estudos que identifiquem os principais medicamentos adjuvantes utilizados e, também, incentiva a atuação e integração da equipe multiprofissional para desenvolver estratégias de intervenção e melhorias a fim de garantir um melhor tratamento farmacoterapêutico e melhor qualidade de vida dos pacientes oncológicos.

\section{Referências}

1. Silva RCF, Hortale VA. Cuidados paliativos oncológicos: elementos para o debate 
de diretrizes nesta área. Cad. Saúde Pública 2006; 22 (10): 2055-66.

2. Diniz RW, Gonçalves MS, Bensi CG, Campos AS, Giglio A, Garcia JB, et al. O conhecimento do diagnóstico de câncer não leva à depressão em pacientes sob cuidados paliativos. Rev. Assoc. Med. Bras. 2006; 52 (5): 298-303, 2006.

3. Instituto Nacional de Câncer (Brasil). Estimativa 2016: Incidência de Câncer no Brasil, Coordenação de Prevenção e Vigilância. Rio de Janeiro: INCA, 2016. Ministério da Saúde. Coordenação de Prevenção e Vigilância. Instituto Nacional de Câncer José Alencar Gomes da Silva, Coordenação de Prevenção e Vigilância. Disponível em: <http://www.inca.gov.br/estimativa/2016/estim ativa-2016-v11.pdf>

4. Machado SM, Sawada NO. Avaliação da qualidade de vida de pacientes oncológicos em tratamento quimioterápico adjuvante. Texto contexto - enferm. 2008; 17 (4): 750-57.

5. Melgaço TB, Carrera JS, Nascimento DEB, Maia CSF. Polifarmácia e ocorrência de possíveis interações medicamentosas. Rev. Para. Med.2011; 25 (1). Disponível em: <http://files.bvs.br/upload/S/01015907/2011/v25n1/a2585.pdf>

6. Barbosa JAA, Belém LF, Sette IMF, Carmo ES, Pereira GJS, Silva EDS. Farmacoterapia Adjuvante no Tratamento da dor oncológica. R. Bras. em Promoção da Saúde. 2008; 21 (2)11220.

7. Instituto Nacional do Câncer (INCA). Cuidados Paliativos, 2014. Brasil. Ministério da Saúde. Disponível em: <http://www1.inca.gov.br/conteudo_view.asp?! $\mathrm{D}=474>$

8. Francisco MFR. Abordagem não farmacológica no controle das náuseas e vômitos induzidos pela quimioterapia. Onco News, 2008; 2 (6) 12-16.

9. Rouquayrol, M. Z. Epidemiologia analítica. In:___. Epidemiologia e Saúde. 3. ed. Rio de Janeiro: Medisi, 1988. 169-203.

10. Jekel JF, Elmore JG, Katz DA. Delineamentos comuns de pesquisa usados em epidemiologia. In: __ Epidemiologia, Bioestatística e Medicina Preventiva. Artes Médicas, Porto Alegre - RS, 1999, 79-87.

11. Santichi EC, Benute GRG, Juhas TR, Peraro EC, Lucia MCS. Rastreio de sintomas de ansiedade e depressão em mulheres em diferentes etapas do tratamento para o câncer de mama. Psicol. hosp., 2012; 10(1), 42-67.

12. Marmo MCR, Caran EMM, Puty FCB, Morais MB. Avaliação do hábito intestinal em pacientes com câncer que utilizam morfina para o controle da dor. Rev. Dor.2012; 13 (3): 243-48.

13. Rafihi-Ferreira R, Soares MRZ. Insônia em pacientes com câncer de mama. Estudos de Psicologia 1, 2012; 29 (4): 597-607.

14. Agra G, Fernandes MA, Platel ICS, Barros NCB, Freire MEM. Constipação em pacientes com doença oncológica avançada em uso de opioides. O mundo da Saúde. 2013; 37 (4): 472-78.

15. Moraes TM, Pimenta. Constipação intestinal em doentes com doença oncológica avançada. Mundo Saúde. 2003; 27 (1): 118-23.

16. Bottino SMB, Fráguas RF, Gattaz WF. Depressão e câncer. Rev. Psiq. Clín.2009; 36 (3): 109-15.

17. Trancas B, Cardoso G, Luengo A, Vieira C, Reis D. Depressão no doente oncológico Considerações diagnósticas e Terapêuticas. Acta, Med. Por. 2010; 23: 1101-12.

18. Santos J, Mota DDCF, Pimenta CAM. Comorbidade fadiga e depressão em pacientes com câncer colo-retal. Rev. Esc. Enferm. 2009; 43 (4): 909-14.

19. Rabelo ML, Borella MLL. Papel do farmacêutico no seguimento farmacoterapêutico para o controle da dor de origem oncológica. Rev. Dor. 2013; 14 (1): 58-60.

20. Corrêa PH, Shibuya E. Administração da Terapia Nutricional em Cuidados Paliativos. Rev. Bras. Cancerol. 2007; 53 (3)317-23.

21. Souza, D. S. Tratamento Farmacológico da Dor em pacientes com Cuidados Paliativos. Prata da casa, 3: escritas do cotidiano de uma equipe que cuida. 2010; 1: 46-52.

22. Pulido JZ, Aleixo SB. Antieméticos em Oncologia. Rev. Bras. Oncologia Clínica. 2004; 1 (3): 35-40.

23. Becker J, Nardin JM. Utilização de antieméticos no tratamento antineoplásico de pacientes oncológicos. R. Bras. Farm. Hosp. Serv. Saúde. 2011; 2 (3): 18-22.

24. Silva, FM. Dispepsia: caracterização e abordagem. Rev. Med. 2008; 87 (4): 213-23.

25. Instituto Nacional do Câncer (INCA). O câncer e seus fatores de risco: o que a educação pode evitar? Ilustrações de Ziraldo - 2 ed. Brasil. Ministério da Saúde. Rev. atual - Rio de Janeiro RJ, 2013.

\section{Endereço para Correspondência}

Universidade Federal da Bahia - UFBA

Campus Anísio Teixeira

e-mail: kelle.oliveira@gmail.com

Recebido em 19/08/2016

Aprovado em 09/03/2017

Publicado em 10/05/2017 